\title{
Metal solidification micro-structure simulation of direct laser deposition based on cellular automation
}

\author{
Tingting Guan ${ }^{a, *}$, Suiyuan Chen ${ }^{b}$, Yue Zhou', Tong Cuid , Jing Liang ${ }^{e}$, Changsheng Liu ${ }^{f}$ \\ Key Laboratory for Anisotropy and Texture of Materials(Ministry of Education), School of Material Science \\ and Engineering, Northeastern University, Shenyang 110819, China \\ atingtinguan@sina.com, bchensy@atm.neu.edu.cn, cyuezhou93@sina.com \\ dcui t@smm.neu.edu.cn, ${ }^{\mathrm{e}}$ Liang j@atm.neu.edu.cn, ${ }^{\mathrm{f}}$ csliu@mail.neu.edu.cn
}

Keywords: Direct laser deposition, non-equilibrium solidification, micro-structure, cellular automation

\begin{abstract}
In recent years, by combining laser cladding, direct laser deposition (DLD) forming as a additive manufacturing (AM) technology has been developed to fabricate high-performance metal components directly. Since the non-equilibrium solidification process involves the thermal and fluidic phenomena inherent which impact the micro-structure, and thus determine the final properties of parts. The mechanism about thermodynamics and dynamics of micro-structure evolution is still not clear during the DLD process. Based on the situation, the article provides an overview of a more mainstream cellular automation (CA) method in the simulation of micro-structure evolution of DLD, briefly describes the basic principle and thought of CA, gives classical and modified models and applications of CA in micro-structure simulation in current research. Meanwhile, several major challenges are also analyzed and the future directions are forecasted.
\end{abstract}

\section{Introduction}

Direct laser deposition (DLD), belongs to a typical laser addictive manufacturing (AM) process, can be utilized to generate complex parts through the addition of multicomponent powder and "rapid melting-solidification "in the melt pool by high-energy laser beam [1], as shown in Fig.1. It has some outstanding characteristics, such as "short process, integration, green", compared with the traditional modification and manufacturing technique. The domestic nuclear industry (China Strategic Emerging Industry), as a representative of " Made in China ", puts forward to use DLD for the nuclear power emergency diesel engine crankshaft (12CrNi2 alloy steel) addictive manufacturing in order to save production cost instead of using traditional casting and forging, while improving the comprehensive mechanical properties, including: toughness, fatigue resistance and wear resistance of alloy steel materials. Since the performance depends on the micro-structure, it's vital to refine micro-structure via DLD.

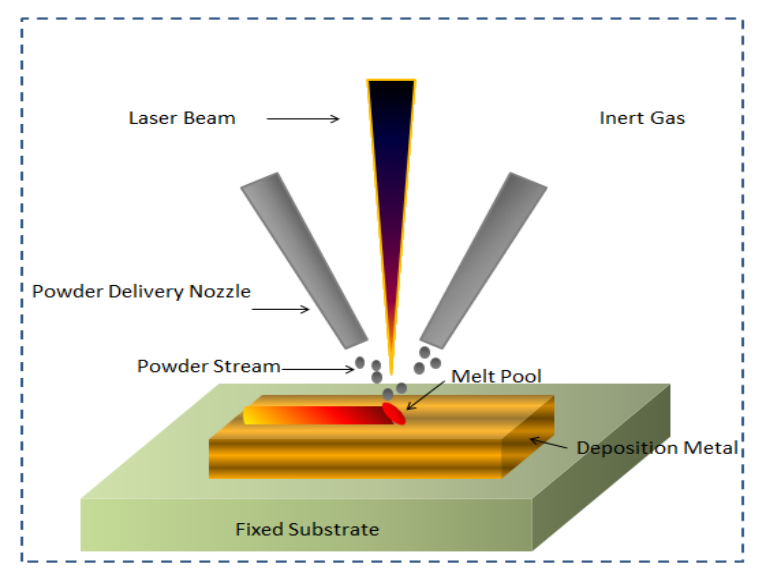

Fig. 1 Direct laser deposition (DLD) shaping schematic 
From 'deposition-to-part', there are many thermal and fluidic phenomena along with superheating and solidification, including: heat transfer, mass transfer and fluid in melt pool. Since the thermal and fluidic behavior provide significant into the final micro-structure and the properties of parts, understanding and optimizing the DLD process requires mastery of the thermal and fluidic phenomena occurring during the build [2]. Current research has made great progress in optimization of parameters, design of materials and evaluation of molding quality. The mechanisms about thermodynamics and dynamics of micro-structure evolution are still not clear, thus needed to be further studied.

With the development of the computer simulation technology in recent years, cellular automation (CA) is one of the methods to simulate the solidification micro-structure, with a fast computation speed and a large area compared with others, Based on the numerical model of thermal/fluidic theoretic, CA can simulate the formation and growth of dendritic, and thus illustrate the evolution mechanism, gradually be applied in simulation of micro-structure evolution during the rapid solidification in DLD. This paper briefly describes the basic principle and thought of CA, gives an overview of the numerical models and applications of CA in micro-structure (growth of dendritic) simulation in current research. Finally, several major challenges are also analyzed and the future directions are forecast.

\section{The basic idea of CA method in solidification}

CA model as a kinetic and stochastic model is scattered in time, space and state. Based on the solidification thermodynamic and growth kinetics, it can determine the locations of the nucleation and crystallization orientation to simulate the growth of dendritic in melt pool. Since the finite element method (FE) is widely used in the field of material molding, cellular automation coupled with Finite Elements method (so-called CAFE model) offers the advantage of providing a direct view of the grain structure (columnar and equiaxed) on the macro-micro scale [3]. Some macroscopic physical quantities, including the temperature field, the solute field and other macroscopic physical quantities of melt pool, is calculated by macroscopic scale mesh and time step. And then continue to be divided into smaller size and regular micro-nodes, which constitute a micro-scale mesh in a partitioned macro mesh, in which using the CA method to simulate the nucleation and growth evolution of micro-structure.

\section{Numerical model of process}

\subsection{Heat transfer and solute diffusion model}

The powder is melted and deposited by a high-energy laser beam to form a melt pool on the substrate. And the energy transfer from the melt pool to the bottom and surrounding quickly. The temperature field of melt pool changes in time and space, which belongs to three-dimensional transient temperature field. The model is defined as follows [4]:

$$
\rho C_{p} \frac{\partial T}{\partial t}=\lambda \frac{\partial^{2} T}{\partial x^{2}}+\lambda \frac{\partial^{2} T}{\partial y^{2}}+\lambda \frac{\partial^{2} T}{\partial z^{2}}+\dot{\Phi}
$$

Where $T$ is the temperature; $t$ is the heat transfer time; $\mathrm{x}, \mathrm{y}$ and $\mathrm{z}$ are space coordinates; $\lambda, \rho$ and $C_{P}$ respectively are coefficient of the heat conductivity, density of the material and specific heat Фcapacity;

is the intensity of internal heat source from phase transition during the solidification of the melt pool, as shown:

$$
\dot{\Phi}=H(T)=\int_{0}^{T} c d T+L\left(1-f_{s}\right)
$$

Where $H(T)$ is enthalpy; $L$ is the latent heat; $f_{s}$ is the solid fraction.

The solute diffusion equation is obtained by $C^{*}{ }_{s}=k \cdot C^{*}{ }_{l}$ (solute partition) of the liquid/solid interface, as shown: 


$$
\frac{\partial C_{i}}{\partial t}=D_{i} \nabla^{2} C_{i}+C_{i}(1-k) \frac{\partial f_{s}}{\partial t}
$$

Where $C_{i}$ and $D_{i}$ respectively are the solute concentration and solute diffusion; $t$ is the time; $k$ is the partition coefficient; $i$ indicates solid or liquid; $C^{*}{ }_{s}$ and $C^{*}{ }_{l}$ are the interface solute concentrations in the solid/liquid phase, respectively, and $f_{s}$ is the solid fraction.

The interface curvature of a cell with solid fraction $f_{s}$ can be obtained by counting the nearest and second-nearest neighboring cells [5], as shown:

$$
K=\frac{1}{l_{C A}}\left(1-2 \frac{f_{s}+\sum_{j}^{N} f_{s}(j)}{N+1}\right)
$$

Where $l_{C A}$ is the length of the CA cell side, $N$ is the number of the nearest and the second-nearest neighboring cells, and $f_{s}(j)$ is the solid fraction of neighboring cells.

At present, Interface curvature calculated by using solid phase fraction is inaccurate because of the error in numerical calculation process. CA-FE method is unable to calculate the partial derivative of the solid-phase fraction accurately. In order to calculate the partial derivative of solid phase fraction, Wei Lei et al. [6] used the method of fluid mechanics on the volume of fluid for calculating the partial derivative, improving the calculation method of interface curvature, the process of adding powder was ignored during the calculation process of DLD. CA-FE method was used to simulate the process from unsteady interface to unsteady solidification of cell/dendrites of solid/ liquid interface of $\mathrm{Fe}-0.1 \mathrm{wt} \% \mathrm{C}$ alloy laser melting process melt pool, and the influence of different crystal orientations on micro-structure of melt pool was analyzed.

\subsection{Nucleation model}

In DLD process, the hetterogeneous nucleation model is adopted to simulate the nucleation evolution of the melt pool. The continuous nucleation is described by Gaussian distribution, $d n / d\left(\Delta T^{\prime}\right)$ is used to describe the grains density. The parameters, including maximum nucleation density $n_{\max }$, the mean undercooling $\Delta T_{N}$, and the standard deviation of the grain density distribution $\Delta T_{\sigma}$. The total density of nuclei $n(\Delta T)$ at a certain undercooling $\Delta T$ is given as follows:

$$
n(\Delta T)=\int_{0}^{\Delta T} \frac{d n}{d\left(\Delta T^{\prime}\right)} d\left(\Delta T^{\prime}\right)=\int_{0}^{\Delta T} \frac{n_{\max }}{\Delta T_{\sigma} \sqrt{2 \pi}} \exp \left[-\frac{1}{2}\left(\frac{\Delta T^{\prime}-\Delta T_{N}}{\Delta T_{\sigma}}\right)\right] d\left(\Delta T^{\prime}\right)
$$

\subsection{Growth model}

KGT model [7] was adopted to calculate the growth process of dendrite tip. Undercooling is the most important factor in the columnar and dendrite growth rate and grain size. The undercooling of the dendritic tip consists of four parts: solute undercooling $\Delta T_{c}$, thermal undercooling $\Delta T_{l}$, kinetics undercooling $\Delta T_{k}$, and curvature undercooling $\Delta T_{r}$. The total undercooling $\Delta T$ is given as follows:

$$
\Delta T=\Delta T_{c}+\Delta T_{l}+\Delta T_{k}+\Delta T_{r}
$$

The kinetic unercooling is not very highly relative to rapid solidification of dendrite tip, so it can be neglected. Most metallic alloys in DLD process neglect thermal undercooling $\Delta T_{l}$ to simplify the model, only consider $\Delta T_{c}$ and $\Delta T_{r}$, so total undercooling $\Delta T$ can be calculated as follows:

$$
\Delta T=m C_{0}\left[1-\frac{1}{\Omega(1-k)}\right]+\frac{2 \Gamma}{R}
$$

Where $m$ is the liquidus slope; $C_{0}$ is the solute concentration in the liquid far from the solid-liquid interface; $k$ is the solute partition coefficient; $\Omega$ is the solute oversaturation during the solidification; $\Gamma$ is the Gibbs-Thomson coefficient; and $R$ is the radius of the dendritic tip. 
The results proved that the nucleation and growth of micro-structure of single melt pool was simulated based on CA-FE. Grain with slightly tilting posture of bulky columnar crystal of melt pool was given priority to form, growth direction and the moving direction of heat source to keep certain angle, most of the grain grew in the opposite direction to the temperature gradient along the melt pool.

J. W. Zhang et al. [8] considered the thermal undercooling $\Delta T_{l}$ and used FE to simulate the temperature field of multi-layer titanium alloy deposited under relatively coarse mesh scale and used the interpolation algorithm to make the temperature field match with the CA model. During the deposition cooling process, the model predicted the thermal history of the laser direct deposition process and the evolution of grain morphology.The formula of total undercooling $\Delta T$ is as follows:

$$
\begin{gathered}
\Delta T=m C_{o}\left[1-\frac{1}{1-(1-k) I\left(P_{c}\right)}\right]+\theta_{t} I\left(P_{t}\right)+\frac{2 \Gamma}{R} \\
R=\left[\frac{\Gamma}{\sigma^{*}\left(m G_{c}^{*}-G^{*}\right)}\right]^{1 / 2}
\end{gathered}
$$

Where $P_{c}$ and $P_{t}$ are the thermal and solutal Peclet numbers, respectively; $\theta_{t}$ is the unit thermal undercooling; and $R$ is the radius of the dendritic tip; $\sigma^{*}$ is the marginal stability constant; and $G_{C}{ }^{*}$ are the effective temperature gradient and concentration gradient, respectively.

\section{Coupling principle of CAFE}

According to the DLD experiment, considering the division and calculation of the model mesh in the simulation, the physical model of the shape is shown in Fig.2:

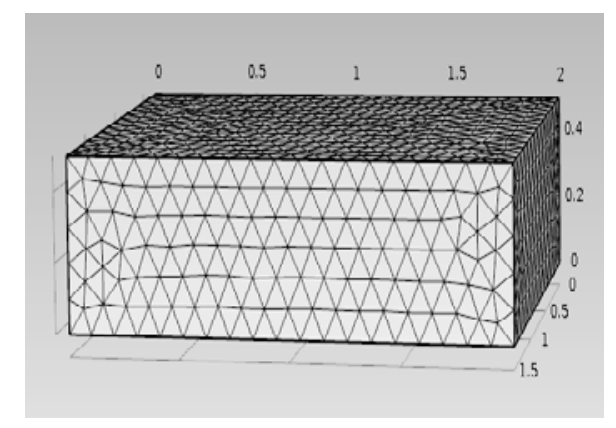

(a)

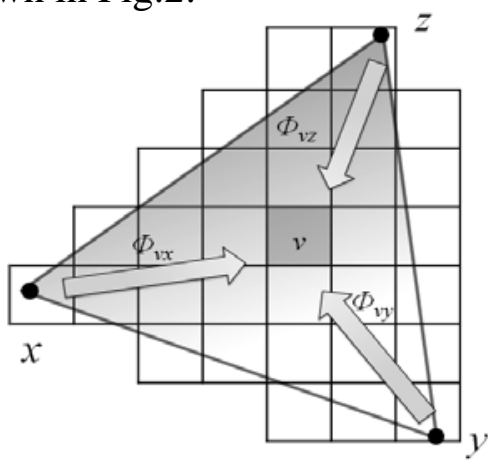

(b)

Fig. 2 (a)The triangular mesh division of physical model; (b) The three linear interpolation coefficients from FE nodes $x, y, z$ to CA cells $v$ are $\Phi_{v x}, \Phi_{v y}, \Phi_{v z}$

In order to couple the CA and FE calculations and simulate the growth of the grain as a function of the thermal field. Using the triangular mesh division of physical model. Based on the result of the macroscopic finite element, the cellular automation (CA) cells, which are square mesh with smaller sizes, perform the calculations to simulate the growth of the grains during DLD. Three nonzero interpolation coefficients $\Phi_{v x}, \Phi_{v y}$ and $\Phi_{v z}$ are defined between nodes of the CA cells and FE mesh. $V$ is considered as a cell unit [9]. The center of the finite element has three nodes: $\mathrm{x}, \mathrm{y}$ and $\mathrm{z}$, as shown in Fig. 2. The nonzero interpolation coefficients can determine the temperature of the CA cell based on the surrounding three finite element nodes. H. Yin et al. [10] neglected the mesh anisotropy used CA-FE method to simulate the two-dimensional region micro-structure evolution of the DLD process (Fe-0.13wt.\%C alloy), and investigated the effect of forming process parameters on the spacing of coagulation dendrites in melt pool. The evolution behavior of the organization is often affected by the anisotropy of the mesh, thus restricting the accuracy of the prediction of CA model. L. Wei et al. [11] used adaptive mesh technology (limited neigh-bor solid fraction, LNSF) which can reduce mesh anisotropy and modified the previous model. 


\section{Conclusions}

(1) DLD is a non-equilibrium rapid solidification process. The dendrite tip growth behavior is in addition to controlled by the diffusion of the solute, it must also consider the solute distribution coefficient with the temperature and the change of dendrite growth, diffusion coefficient, along with the change of temperature and the influence of curvature and dynamic effect across the undercooling.

(2) Most of the CA method in the micro-structure simulation of melt pool is based on two-dimensional simulation. In order to simulate the micro-structure evolution of the melt pool visually, The three dimensional CA model is needed in the future.

(3) Material is diversified gradually in DLD, the CA method needs to break through the bondage of the field for its application. It should be widely used in steel materials simulation to lay the theoretical basis of alloy steel material during the additive manufacturing.

\section{Acknowledgements}

This work was financially supported by National Key R\&D Program of China (2016YFB1100201), National Natural Science Foundation of Liaoning United fund (U1508213).

\section{References}

[1] Z. T. Gan, G. Yu, X. L. He, S. X. Li, International Journal of Heat and Mass Transfer. 104 (2017) 28-38.

[2] S. M. Thompson, L. K. Bian, N. Shamsaei, A. Yadollahi, Additive Manufacturing. 8(2015) 36-62.

[3] M. Rappaz, Current Opinion in Solid State and Materials Science. 20 (2016) 37-45.

[4] J.C. Li, Calculation of Diffraction and Thermal Effect of Laser Science Press, Beijing. (2002).

[5] L. Nastac, Acta Mater. 47 (1999) 4253-4262.

[6] L. Wei, X. Lin, M. Wang, W. D. Huang, Acta Phys Sin. 64 (2015) 1-6.

[7] M. A. Zaeem, H. B. Yin, S. D. Felicelli, Journal of Materials Science \& Technology. 28 (2012) 137-146.

[8] J. W. Zhang, F. Liou, S. William, Additive Manufacturing. 11(2016) 32-39.

[9] F. J. Tian, Z. G. Li, J. X. Song, Journal of Alloys and Compounds. 676 (2016) 542-550.

[10] H. Yin, S .D. Felicelli, Acta Materialia. 58 (2010) 1455-1465.

[11] L. Wei, X. Lin, M. Wang, W. D. Huang, Physica B: Condensed Matter. 407 (2012) 2471-2475. 